\title{
Diagnóstico microbiológico de las infecciones de transmisión sexual (ITS). Parte 1. ITS no virales
}

\author{
M. Angélica Martínez T.
}

\section{Microbiological diagnosis of sexually transmitted infections (STI) Part 1. Non-viral STI}

Non-viral sexually transmitted infections (STI) are an important cause of physical, psychological and social distress, have severe consequences for women's reproductive health and may be transmitted to the newborn child. These infections are also risk factors for the acquisition and transmission of HIV and other STI, and for premature labor. In the last years we have observed a gradual decrease in the national incidence of gonorrhea. The implementation of a screening program in our country for Chlamydia trachomatis is necessary, since up to $80 \%$ of infections in women are asymptomatic. Due to medical, psychosocial and legal reasons, laboratory diagnosis of STI has to be certain. This offers a great challenge to laboratories. Since etiological agents are susceptible to environmental conditions, present a high adaptation to their human host and have particular physiological characteristics, their laboratory diagnosis is more difficult than diagnosis of conventional microorganisms. Otherwise, the diagnostic techniques currently available for non-viral STI are characterized by their excellent sensitivity and specificity, which result of great interest given the curable nature of these infections. Clinical specimens obtained for diagnosis of STI and other genital infections, such as bacterial vaginosis or Candidiasis represent a large proportion of specimens processed by clinical laboratories. Thus, the creation of norms and quality control guidelines for laboratories which diagnose these infections, and also the epidemiologic and genetic surveillance of circulating sex transmitted microorganisms should be considered a priority in our country. The objective of this study is to review current literature on accurate diagnostic procedures especially for three non-viral STI agents: C. trachomatis, N. gonorrhoeae, and Trichomonas vaginalis.

Key words: Laboratory diagnosis, non-viral sexually transmitted infections.

Palabras clave: Diagnóstico microbiológico, infecciones de transmisión sexual, no-virales.

\section{Introducción}

$\mathrm{L}$ as infecciones de transmisión sexual (ITS) no virales son causa de sufrimiento físico, psicológico y social, tienen consecuencias graves para la salud reproductiva de la mujer y pueden ser transmitidas al recién nacido ${ }^{1}$. Estas infecciones son también un factor de riesgo para adquirir y transmitir otras ITS y en las mujeres embarazadas están asociadas con riesgo de parto prematuro ${ }^{1}$. En los últimos años se ha producido una declinación en las tasas nacionales de gonorrea, pero es necesario implementar el tamizaje de Chlamydia trachomatis, dada la ineficiencia del manejo sindrómico de flujo vaginal para la infección por esta bacteria, fundamentalmente asintomática ${ }^{2-3} . \mathrm{El}$ manejo sindromático tiene también limitaciones para el diagnóstico de tricomoniasis, al menos en mujeres gestantes ${ }^{4}$.

Por razones médicas, psicosociales y legales, el diagnóstico de laboratorio de las ITS debe ser de certeza, lo que plantea un desafío al laboratorio, ya que la labilidad de los agentes, su gran adaptación al hospedero humano y las particularidades de su fisiología hacen que su diagnóstico presente mayor dificultad que el de otras bacterias ${ }^{5}$. Sin embargo, las técnicas de laboratorio disponibles actualmente para el diagnóstico de las ITS no virales han alcanzado una gran eficiencia, lo que resulta muy valioso dada la naturaleza curable de estas ITS.

Las muestras clínicas para diagnóstico de ITS e infecciones genitales significan un alto porcentaje de las muestras clínicas recibidas en los laboratorios clínicos. Sumado a las variaciones genéticas frecuentes que experimentan sus agentes etiológicos y que afectan al diagnóstico microbiológico hacen prioritarios la vigilancia epidemiológica y genética de las cepas que circulan en el país y el establecimiento de normas de control de calidad de los procedimientos de diagnóstico de laboratorio ${ }^{6-8}$.

Esta revisión se centra en tres microorganismos de transmisión sexual; dos bacterianos, C. trachomatis y Neisseria gonorrhoeae y en Trichomonas vaginalis, y
Facultad de Medicina, Universidad de Chile, Santiago

Programa de Microbiología y Micología, ICBM

Recibido: 21 de abril de 2009 Aceptado: 31 de julio de 2009

La autora no tiene conflictos de interés

Correspondencia a: M. Angélica Martínez Tagle mamartin@med.uchile.cl 
tiene como objetivos mostrar las evidencias que justifican la implementación de técnicas de diagnóstico eficientes para estos agentes y el conocimiento que apoya las recomendaciones actuales de diagnóstico microbiológico.

\section{Aspectos relacionados con la patogenia e impacto clínico de los agentes}

Chlamydia trachomatis es la bacteria de transmisión sexual más frecuente en el mundo, estimándose en alrededor de 90 millones los casos nuevos cada año ${ }^{3}$. Entre 70 y $75 \%$ de las infecciones en mujeres son asintomáticas, lo que dificulta su detección y tratamiento, pudiendo persistir por meses o años ${ }^{3}$. Morré y cols ${ }^{9}$, analizaron el curso de infecciones asintomáticas por este agente en una cohorte de un año de duración, que incluyó 30 casos y 186 controles. Solamente 44,7\% de las mujeres infectadas eliminaron la clamidia durante el año de observación. Se ha calculado que 10 a $40 \%$ de los casos de infección cervical resultan en una enfermedad inflamatoria pelviana (EIP) ${ }^{10}$. El riesgo más temido de la EIP es la infertilidad secundaria a la obstrucción tubaria. Chlamydia trachomatis es la causa más frecuente de EIP en todo el mundo. En E.U.A. se ha aislado en 25 a $60 \%$ de las EIP y en Suecia entre 40 y $60 \%$. En Chile representó el $28 \%$ de las pacientes hospitalizadas por EIP aguda ${ }^{11}$. Chlamydia trachomatis es una causa importante de conjuntivitis y neumonía neonatal en los países donde no se efectúa tamizaje de la bacteria durante el embarazo ${ }^{12,13}$. Se ha estimado que 8 a $44 \%$ de neonatos expuestos al nacer desarrollan conjuntivitis y 0 a $17 \%$ neumonía ${ }^{14}$. En Chile, Valencia y cols investigaron la participación de C. trachomatis en conjuntivitis neonatal, detectando mediante reacción de polimerasa en cadena (RPC), 7,8\% de casos positivos, mientras que Martínez y cols asociaron también por RPC a C. trachomatis como etiología de $18,1 \%$ de las neumonías del primer trimestre sugerentes de clamidia ${ }^{15,16}$.

Neisseria gonorrhoeae es el agente etiológico de la gonorrea, una de las ITS de mayor prevalencia en el mundo, con más de 60 millones de casos reportados anualmente 3 . Neisseria gonorrhoeae es una de las bacterias que posee el mayor arsenal de factores de virulencia y estrategias para la evasión de la respuesta inmune del ser humano. Una característica notable de este microorganismo es su capacidad de revertir la expresión de sus moléculas de superficie, o experimentar variación antigénica con alta frecuencia; destacan por su variabilidad las fimbrias, las proteínas de opacidad de colonias (Opa) y el lipooligosacárido (LOS), lo cual ha retardado el desarrollo de vacunas ${ }^{17}$. A diferencia de la intensa respuesta inflamatoria que causa $N$. gonorrhoeae en la uretra masculina, 50 a $80 \%$ de las infecciones cervicales son asintomáticas y entre 70 y $90 \%$ de las mujeres con infección gonocóccica diseminada no tienen signos de infección genital ${ }^{18}$. La adherencia de $N$. gonorrhoeae a las células cervicales no induce una respuesta inflamatoria humoral ni tampoco induce la expresión de las citoquinas pro-inflamatorias IL-1, IL-6 e IL-8, lo que facilita su sobrevida en el cuello uterino, logrando un estado similar al de portación ${ }^{18}$. Infección genital ascendente se presenta en $20 \%$ de las mujeres con gonorrea cervical. El ascenso es favorecido probablemente por factores hormonales que inducen cambios en la expresión de receptores en la mucosa genital y favorecen la adherencia de la neiseria. En consecuencia con esta hipótesis, destaca la mayor frecuencia de casos de EIP e infección gonocóccica diseminada (IGD) durante la etapa menstrual ${ }^{19}$. Similar a C. trachomatis, N. gonorrhoeae puede ser adquirida por el neonato al pasar por el canal del parto. La infección suele manifestarse como una conjuntivitis que, de no ser tratada adecuadamente, puede conducir a la ceguera.

La infección por $T$. vaginalis es la ITS no viral más frecuente en el mundo, con $\sim 173$ millones de casos nuevos cada año ${ }^{3}$. En la mujer, el microorganismo infecta principalmente el epitelio escamoso de la vagina dando origen a vulvovaginitis de diversa intensidad, pero 25 a $50 \%$ de las mujeres infectadas son asintomáticas ${ }^{20}$. Trichomonas vaginalis es un factor de riesgo para rotura prematura de membranas y de parto prematuro en mujeres embarazadas $^{21,22}$. La tricomoniasis constituye la etiología de menos de $5 \%$ de los casos de uretritis masculina ${ }^{20}$. Sin embargo, sólo $47 \%$ de los hombres infectados presenta secreción uretral y $22 \%$ disuria, constituyendo por ello un reservorio de infección para la mujer ${ }^{23}$. Por otra parte, utilizando como referencia el cultivo o una técnica de RPC confirmada, Hobbs y cols demostraron que 73,2\% de 287 hombres, parejas de mujeres con tricomoniasis, estaba infectado, demostrando la alta concordancia de la infección entre parejas sexuales ${ }^{24}$. Como ocurre con otras ITS, la tricomoniasis es un factor de riesgo para la adquisición y transmisión de la infección por VIH y es un marcador significativo de infección concomitante con otros agentes de transmisión sexual, incluyendo $C$. trachomatis y $N$. gonorrhoeae ${ }^{25}$.

\section{Diagnóstico microbiológico}

\section{Chlamydia trachomatis}

Existen numerosos métodos para el diagnóstico de C. trachomatis, los cuales podemos clasificar en procedimientos de cultivo celular, técnicas de detección de antígenos, métodos de hibridación de ácidos nucleicos y técnicas de amplificación de ácidos nucleicos (TAAN). Estos procedimientos tienen distintos requerimientos de toma y transporte de muestras, en relación con las características de las técnicas a utilizar. 


\section{Muestras clínicas}

Las recomendaciones del CDC para diagnóstico de infección cervical, efectuado por un procedimiento de TAAN, incluían en el año 2002 solamente las muestras de secreción endo-cervical u orina de primer chorro $^{26}$. Con posterioridad, la FDA aprobó la muestra vaginal por el ensayo comercial Gen-Probe APTIMA AC2. No existen diferencias significativas entre el rendimiento de las muestras endo-cervicales u orina, incluidas las muestras de mujeres asintomáticas, para los distintos métodos de TAAN $^{27}$. En los últimos años se han acumulado también evidencias que las muestras de secreción vaginal tienen mayor rendimiento que las de orina para los procedimientos de TAAN en mujeres ${ }^{28,29}$. La toma de muestra vaginal tiene muy buena aceptación entre las adolescentes y es menos invasora que la muestra cervical por lo que cuando no es necesario efectuar examen pélvico, constituiría la toma de muestra de elección. Para diagnóstico por un procedimiento distinto a las técnicas de TAAN, el CDC recomienda solamente el empleo de muestras endocervicales, ya que la sensibilidad de estos procedimientos en orina de primer chorro es inferior ${ }^{26}$. Para el diagnóstico de infección uretral en hombres, el CDC recomienda las muestras de secreción endo-uretral u orina de primer chorro para diagnóstico por TAAN y la muestra endo-uretral solamente para otros procedimientos de diagnóstico ${ }^{26}$. El diagnóstico de infección faríngea y de proctitis se efectúa en muestras faríngea y rectal respectivamente, estando indicado su estudio solamente por las técnicas de cultivo celular o de IFD con anticuerpos monoclonales (AcMo) anti Omp1 ${ }^{26}$. En la Tabla 1 se hace un esquema de las muestras indicadas y los procedimientos recomendados para el diagnóstico de C. trachomatis.

Con respecto a los procedimientos para la toma de muestra, se ha establecido que, con la excepción de las muestras para cultivo celular, las muestras clínicas para el resto de los procedimientos deben ser obtenidas siguiendo estrictamente las indicaciones del fabricante del kit, como asimismo respetando los materiales de toma de muestra; tipo de tórula y/o cepillo y medio de transporte ${ }^{26}$. Esto se debe a que los valores de sensibilidad y especificidad indicados para la técnica por el fabricante han sido obtenidos en esas condiciones. No obstante, podemos hacer algunas generalizaciones: la toma de muestra uretral debiera obtenerse, al menos, una hora post micción. Se recomienda obtener en primer lugar las muestras destinadas a diagnóstico de $N$. gonorrhoeae y con posterioridad las necesarias para clamidia, y es necesario retirar las secreciones purulentas en uretra o cérvix previo a la toma de muestras ${ }^{26}$.

\section{Técnicas de diagnóstico}

Cultivo celular. El aislamiento de C. trachomatis en líneas celulares es el procedimiento de mayor especifici-
Tabla 1. Muestras clínicas de elección para diagnóstico de C. trachomatis de acuerdo al procedimiento de diagnóstico empleado ${ }^{26}$

\begin{tabular}{|lll|}
\hline Tipo de infección & $\begin{array}{l}\text { Muestra clínica de } \\
\text { elección }\end{array}$ & $\begin{array}{l}\text { Técnica de diagnóstico } \\
\text { apropiada }\end{array}$ \\
\hline Infección cervical & $\begin{array}{l}\text { Hisopado endo-cervical } \\
\text { Hisopado vaginal } \\
\text { Orina de primer chorro } \\
\text { Hisopado endo-cervical }\end{array}$ & TAAN \\
\hline Infección uretral & $\begin{array}{l}\text { Hisopado endouretral } \\
\text { Orina de primer chorro }\end{array}$ & TAAN IFD \\
\hline Infección faríngea & Hisopado endo-uretral & Cultivo, EIA, IFD \\
\hline Infección rectal & Hisopado faríngeo & Cultivo, IFD (Omp1) \\
\hline Conjuntivitis adultos & Hisopado rectal & Cultivo, IFD (Omp1) \\
\hline Conjuntivitis neonatal & Hisopado conjuntival & Cultivo, IFD (Omp1) \\
\hline Neumonía del primer trimestre & Hisopado conjuntival & Cultivo, IFD (Omp1) \\
\hline Sospecha de abuso sexual niñitas & Muestra vaginal & Cultivo, IFD \\
\hline
\end{tabular}

TAAN: técnicas de amplificación de ácidos nucleicos

EIA: enzimo inmuno análisis

IFD: inmunofluorescencia directa

Omp: outer membrane protein

dad para el diagnóstico de clamidia, virtualmente 100\% específico. Por este motivo, es la técnica de elección en casos de sospecha de abuso sexual, en los cuales hay graves implicancias médico-legales. No obstante, por su baja sensibilidad, complejidad y los requerimientos de una cadena de frío para la mantención de la viabilidad de C. trachomatis, no es recomendada como técnica de diagnóstico ${ }^{26,30}$.

Técnicas de detección antigénica. Existen dos tipos de procedimientos para la detección de antígenos de $C$. trachomatis: la inmunofluorescencia directa (IFD) con anticuerpos monoclonales y los enzimo inmunoanálisis (EIA). En esta segunda categoría se incluye una gran variedad de formatos como son los ensayos clásicos en microplaca, los ensayos automatizados y las pruebas rápidas.

La IFD es una técnica rápida de diagnóstico pero la observación microscópica es demandante y por ello no adecuada para el procesamiento contínuo de un gran número de muestras clínicas. En los países desarrollados donde el personal calificado es caro, se utilizan preferentemente las técnicas de mayor automatización como 
EIA y las técnicas moleculares. La IFD se basa en el empleo de anticuerpos monoclonales (AcMo) dirigidos a la proteína principal de la membrana externa de $C$. trachomatis, Omp1, o al LPS, o contienen una mezcla de los dos AcMo. La calidad de la tinción de los ensayos de IFD que contienen AcMo con especificidad por el LPS es inferior, ya que la tinción de los corpúsculos elementales se ve difuminada ${ }^{30}$. Pero, además, algunos AcMo dirigidos al LPS, reconocen el LPS de otras bacterias gramnegativas, por lo que sólo se recomienda el empleo de AcMo anti Omp1 ${ }^{26}$. La fortaleza de la IFD es permitir evaluar la calidad de la muestra. Una muestra inadecuada no contiene células epiteliales columnares, sino que un exceso de mucus, o un predominio de células escamosas vaginales. La sensibilidad de la IFD, en comparación con el cultivo celular, varía entre 80 y $90 \%$, mientras que la especificidad fluctúa entre 94 y $99 \%{ }^{30}$. Los valores más altos de sensibilidad son observados en poblaciones con alta prevalencia de infección por clamidias y en población sintomática $^{31}$. En comparación, en poblaciones con baja prevalencia de infección y en personas asintomáticas, se ha demostrado un escaso número de corpúsculos elementales por lámina, inferior a 10 , lo que reduciría la sensibilidad de la técnica ${ }^{30-32}$. Dada que la sensibilidad del cultivo varía entre 75 y $80 \%$, en comparación con las técnicas de AAN, la sensibilidad de la IFD es aún menor ${ }^{30}$.

Los EIA son métodos de diagnóstico apropiados para laboratorios que reciben una gran cantidad de muestras, ya que son procedimientos automatizados o semi-automatizados. Los EIA clásicos como Chlamydiazyme ${ }^{\circledR}$ (Abbott, USA), Microtrak ${ }^{\circledR}$ (Syva, E.U.A.) y VIDAS ${ }^{\circledR}($ BioMerieux, Francia) incluyen un reactivo de confirmación de las muestras positivas y su especificidad varía actualmente entre 98 y $100 \%$, en comparación con el cultivo celu$\operatorname{lar}^{30,33-35}$. En contraste, la sensibilidad de los EIA es inferior a la del cultivo celular ${ }^{30,33-37}$. Newhall y cols compararon en un estudio multicéntrico, varios procedimientos de diagnóstico inmunológico en el formato de EIA e IFD para la evaluación de 4.980 muestras cervicales, en una población con baja prevalencia de infección ${ }^{36}$. Mientras la especificidad de los procedimientos, luego de efectuar ensayo confirmatorio varió entre 99,6 y $99,8 \%$, las sensibilidades de las técnicas fluctuaron entre 61,9 y $74,5 \%$. La IFD (Microtrak ${ }^{\circledR}$, Syva) fue la técnica más sensible. Entre las técnicas de EIA, EIA Syva fue la más sensible, $70,6 \%$, mientras que EIA Sanofi y EIA Abbott tuvieron valores de sensibilidad de 61,6 y $61,9 \%$ respectivamente. Waites y cols compararon un nuevo EIA, EIAACCESS $\AA$ (Beckman/Sanofi, USA) con la amplificación del ADN mediante reacción de la ligasa en cadena $(\mathrm{LCx} \circledast$, Abbott, USA), actualmente discontinuada, para el diagnóstico de infección cervical, demostrando $84 \%$ de sensibilidad y $99,7 \%$ de especificidad ${ }^{35}$. ACCESS $®$ emplea anticuerpos monoclonales acoplados a partículas magnéticas para lectura mediante quimio-luminiscencia y tiene un ensayo confirmatorio. Los EIA son utilizados frecuentemente en los laboratorios clínicos para el diagnóstico de C. trachomatis en muestras de orina de primer chorro, pero esta muestra sólo se recomienda en ensayos de amplificación. Shafer y cols, evaluaron la utilidad de Chlamydiazyme ${ }^{\circledR}$ confirmada mediante IFD (Microtrak ${ }^{\circledR}$, Syva) para el diagnóstico de $C$. trachomatis en muestras de orina de hombres adolescentes asintomáticos, considerando como técnica de referencia al cultivo celular ${ }^{38}$. En una población con una prevalencia de $7 \%$ de infección, el EIA tuvo $78 \%$ de sensibilidad y $100 \%$ de especificidad ${ }^{38}$. Estos resultados confirman la baja sensibilidad del diagnóstico en muestras de orina.

Las pruebas rápidas no constituyen procedimientos de laboratorio sino que son técnicas diseñadas para emplear en la consulta de atención primaria, como apoyo al diagnóstico clínico y son buenas herramientas para tamizaje a nivel de consulta primaria ${ }^{39}$. Proporcionan además resultados inmediatos lo que a su vez permite tratamiento inmediato, disminuyendo la pérdida de pacientes. Sin embargo, su especificidad es inferior a la de los ensayos convencionales y dado que la prevalencia en laboratorios es baja o moderada, tienen bajo valor predictor positivo ${ }^{40}$.

Ensayos de hibridación con sondas. Existen dos ensayos disponibles: Gen Probe PACE® y PACE2 $®$. Estos ensayos son ampliamente usados en E.U.A., desplazando a las técnicas de detección antigénica, pero en Chile no han tenido aceptación, ya que no tienen ventajas con respecto a su eficiencia diagnóstica y resultan de mayor costo.

Técnicas de amplificación de los ácidos nucleicos. Las TAAN son los procedimientos de elección para el diagnóstico de $C$. trachomatis en virtud de su sensibilidad y especificidad y porque no requieren de la toma de muestras invasoras para su ejecución ${ }^{5,28,41-42}$. Se ha demostrado que las TAAN detectan entre 17 y $28 \%$ más infecciones que otros procedimientos de diagnóstico ${ }^{27,43}$. Existen varias tecnologías comerciales de TAAN, siendo los más conocidos la reacción de polimerasa en cadena (RPC), la reacción de ligasa en cadena (RLC) y la amplificación por desplazamiento de hebra (SDA). Varios ensayos detectan simultáneamente a $C$. trachomatis y $N$. gonorrhoeae. No obstante, con el objeto de evitar resultados falsamente positivos en poblaciones con baja prevalencia de infección, el CDC recomendó en el año 2002 confirmar los ensayos positivos para $C$. trachomatis si el valor predictor positivo (VPP) del procedimiento fuera inferior a $90 \%{ }^{26}$. Esta recomendación ha sido seguida de innumerables horas de discusión y estudio entre los investigadores. Las recomendaciones del CDC se fundamentan en la premisa de que si no se confirma un resultado positivo, es porque corresponde a un falso positivo. Sin embargo, no existen 
evidencias que avalen esta suposición. Schachter y cols, evaluaron las estrategias de confirmación sugeridas por el CDC en distintas tecnologías de TAAN: repetir el procedimiento en la muestra original; reevaluar la muestra clínica con una TAAN distinta; y efectuar una TAAN diferente en un duplicado de la muestra ${ }^{44}$. Las técnicas de RPC: AC2 y ACT ${ }^{\circledR}$ (Gen-Probe) y AMPLICOR ${ }^{\circledR}$ (Roche) confirmaron 96,7 a 99,5\% de los resultados positivos iniciales, con una prevalencia de laboratorio de $5 \%$. Más aún, los procedimientos fueron capaces de confirmar cruzadamente los resultados de los otros ensayos. Los resultados fueron menos afortunados para RLC, LCx ${ }^{\circledR}$ (Abbott) con 90,4\% confirmados y SDA ${ }^{\circledR}$ (Becton Dickinson) con $83,8 \%$ confirmados con una sola repetición y $92,5 \%$ en segunda repetición ${ }^{44}$. Los autores concluyen que la repetición de aquellos ensayos con escaso blanco de amplificación da resultados fluctuantes positivos-negativos debido a la distribución variable de las moléculas blanco en la muestra, lo que no significa que sean falsos positivos. Los resultados también confirman que existen diferencias entre los TAAN, siendo las técnicas de RPC las más sensibles ${ }^{45}$.

La sensibilidad actual de los ensayos de TAAN es excelente; por ello es necesario poner mayor énfasis en los procedimientos de preparación de las muestras clínicas, porque afectan la sensibilidad de los ensayos. Esto es especialmente importante en la ejecución de RPC no comerciales. Es urgente el desarrollo de normas de toma y transporte de muestras a los laboratorios que efectúan RPC caseras, ya que las decisiones de los laboratorios deben estar basadas en evidencias. Los ensayos de TAAN deben incluir como parte de los controles a controles de amplificación que garanticen que la muestra no contiene inhibidores de la reacción, que es el principal problema de las TAAN.

\section{Neisseria gonorrhoeae}

\section{Muestras clínicas}

Las muestras deberían ser obtenidas preferentemente con tórulas de dacrón o de rayón ${ }^{46,47}$. Recientemente se ha demostrado la eficiencia de un nuevo tipo de tórulas fabricadas con nylon ${ }^{47}$. En el caso de usar tórulas de algodón, deberían ser de calidad certificada ya que los ácidos grasos inhiben el crecimiento de la neiseria ${ }^{46,48}$. La siembra inmediata de las muestras clínicas en medios de cultivo ha demostrado ser el procedimiento óptimo para el aislamiento de esta bacteria, pero generalmente esto no es posible de efectuar, siendo esencial el uso de medios de transporte ${ }^{49}$.

Varios factores se deben considerar en el transporte de muestras. Neisseria gonorrhoeae es un microorganismo sensible al frío y la desecación, no crece bien en presencia de microbiota comensal y puede estar además en bajo número en las muestras clínicas. En general, es preferible emplear medios de transporte comerciales ya que han sido evaluados por las normas de referencia del CLSI y comparados con la siembra directa en medios de cultivo; si no es posible, el laboratorio debería efectuar controles de calidad de sus medios de transporte ${ }^{50}$. La mayoría de los medios de transporte comerciales que incluyen carbón activado han demostrado ser superiores a aquellos que no lo contienen, en conservar la viabilidad de $N$. gonorrhoeae, a las 6 y 24 h de conservación de las muestras a temperatura ambiente ${ }^{51}$. El carbón activado elimina los ácidos grasos insaturados, tóxicos para la neiseria, pero su presencia interfiere con la lectura del examen microscópico directo de las muestras clínicas ${ }^{48}$. Algunos estudios han demostrado que el medio de transporte Amies, con y sin carbón activado, de la firma Copan (Copan Diagnostics, Corona, Ca, E.U.A.) tiene igual eficiencia para conservar la viabilidad de cepas clínicas de $N$. gonorrhoeae ${ }^{51}$ y además ha demostrado ser superior a otros medios de transporte comerciales ${ }^{48,51}$. Con respecto al tiempo de mantención de la viabilidad de $N$. gonorrhoeae, un estudio efectuado en medio de transporte Copan permitió la recuperación del $95 \%$ de 466 muestras positivas por siembra directa a las $24 \mathrm{~h}$ de incubación y $98 \%$ de sobrevida a las $6 \mathrm{~h}^{48}$. Cinco de los siete casos en que no se recuperó la neiseria se debieron al crecimiento de levaduras en el medio de transporte, superando con ello la capacidad fungicida del agar Thayer Martin ${ }^{51}$. Esto podría ser crítico en aquellas muestras endo-cervicales con un bajo número de microorganismos. Olsen y cols, demostraron que el número de ufc de $N$. gonorrhoeae presentes en algunas muestras endo-cervicales puede ser tan bajo como $10^{2} \mathrm{ufc} / \mathrm{ml}$, mientras que la concentración de levaduras y de bacterias comensales puede alcanzar $10^{6}$ $\mathrm{ufc} / \mathrm{ml}^{48}$. Recientemente, un estudio observó la sobrevida de $N$. gonorrhoeae a $4{ }^{\circ} \mathrm{C}$ en un medio de transporte de última generación ${ }^{47}$. Si bien el frío inhibiría el sobrecrecimiento de otros microorganismos, el número de cepas evaluadas es muy bajo y se requieren más estudios antes de recomendar la conservación de las muestras a baja temperatura. Los resultados permiten sugerir que, cuando se disponga de medios de transporte de calidad convencional, las muestras sean conservadas a temperatura ambiente e inoculadas antes de las 6 horas de obtenidas.

\section{Técnicas de diagnóstico}

Examen microscópico directo. El examen microscópico directo tiene gran utilidad para el diagnóstico de uretritis gonocóccica masculina. Se basa en la demostración al microscopio de diplococos gramnegativos intra y extracelulares en un extendido de secreción uretral teñido con $\mathrm{Gram}^{52}$. Los pacientes con infección sintomática presentan habitualmente dos o más leucocitos con bacterias intracelulares ${ }^{52}$. La sensibilidad del examen 
directo teñido con Gram en hombres sintomáticos es 90 a $95 \%$ y su especificidad 95 a $100 \%{ }^{49}$. La correlación del Gram de secreción uretral comparado con la detección de $N$. gonorrhoeae mediante hibridación con sondas para el diagnóstico, en hombres con uretritis sintomática, es $99,6 \% 53$. Estos valores confirman la eficiencia de la tinción de Gram para el diagnóstico presuntivo de uretritis gonocóccica en hombres, especialmente en lugares que no cuentan con diagnóstico de laboratorio ${ }^{49}$. La sensibilidad de la tinción de Gram en muestras endo-cervicales es de sólo 50 a $70 \%$, con una especificidad de $90 \%$, por lo que no se recomienda su empleo como método presuntivo ${ }^{49,54}$.

Cultivo. En la Tabla 2 se indican las muestras clínicas de elección para el diagnóstico de $N$. gonorrhoeae mediante cultivo. Esta es la técnica de referencia para el diagnóstico de $N$. gonorrhoeae a partir de muestras clínicas obtenidas de cualquier sitio $^{26}$. Esta recomendación incluye el estudio de muestras endo-cervicales, uretrales, rectales, faríngeas y de sitios estériles de adultos y es válida para infecciones sintomáticas y asintomáticas, como, asimismo, para infecciones purulentas y para aquellas en que no se observa secreción ${ }^{26}$. El cultivo es también la técnica de elección para el estudio de muestras conjuntivales en neonatos y adultos y para el diagnóstico en casos de abuso sexual ${ }^{26}$. Por una parte, el cultivo es un

\begin{tabular}{|c|c|c|}
\hline Tipo de infección & $\begin{array}{l}\text { Muestra clínica } \\
\text { apropiada }\end{array}$ & $\begin{array}{l}\text { Procedimiento } \\
\text { recomendado }\end{array}$ \\
\hline Uretritis (gonorrea) & Secreción uretral & Cultivo \\
\hline Infección uretral asintomática & Hisopado endo-uretral & Cultivo \\
\hline Infección cervical & Hisopado endo-cervical & Cultivo \\
\hline Infección rectal & Hisopado rectal & Cultivo \\
\hline Infección faríngea & Hisopado faríngeo & Cultivo \\
\hline Infección de sitios habitualmente estériles & $\begin{array}{l}\text { De acuerdo al tipo de } \\
\text { infección }\end{array}$ & Cultivo \\
\hline Conjuntivitis adultos y neonatal & Hisopado conjuntival & Cultivo \\
\hline Sospecha de abuso sexual en niñitas & Muestra vaginal & $\begin{array}{l}\text { Cultivo } \\
\text { TAAN confirmado }\end{array}$ \\
\hline Infección uretral masculina & $\begin{array}{l}\text { Secreción endo-uretral u } \\
\text { orina de primer chorro }\end{array}$ & TAAN* \\
\hline Infección endo-cervical & Hisopado endo-cervical & \\
\hline
\end{tabular}

procedimiento bien estandarizado, que posee una buena sensibilidad y especificidad, es más simple y económico de efectuar que las técnicas moleculares y, por otra parte, permite recolectar las cepas circulantes para la vigilancia de la susceptibilidad antimicrobiana de esta bacteria. El cultivo por lo tanto, proporciona un diagnóstico de certeza de infección gonocóccica ${ }^{26}$. No obstante, existen algunas situaciones precisas en que el cultivo puede ser reemplazado por las técnicas moleculares. Una de estas situaciones se presenta cuando se prevén condiciones sub-óptimas de transporte y de conservación de muestras, las que no aseguran la viabilidad de $N$. gonorrhoeae y solamente incluye al estudio de muestras endo-cervicales y a muestras endo-uretrales $u$ orina de primer chorro en hombres ${ }^{26}$. Otra situación de excepción es el diagnóstico de $N$. gonorrhoeae en casos de abuso sexual, cuando no se dispone de cultivo. En esta situación, se recomienda confirmar un resultado positivo por otro procedimiento molecular ${ }^{26}$. En resumen, no se recomienda efectuar diagnóstico molecular de $N$. gonorrhoeae en muestras vaginales y orina en mujeres adultas, secreción del meato urinario en hombres y diagnóstico de infección faríngea e infección rectal, lo cual se explica por una inferior sensibilidad y/o especificidad de las técnicas moleculares con respecto del cultivo en las muestras indicadas.

Medios de cultivo. Neisseria gonorrhoeae crece entre 35 y $36^{\circ} \mathrm{C}$ en una atmósfera húmeda con 3 a $5 \%$ de $\mathrm{CO}_{2}$. El aporte inmediato de una atmósfera enriquecida en $\mathrm{CO}_{2}$ es un factor incluso más crítico que la incubación inmediata a $35-36{ }^{\circ} \mathrm{C}^{49}$. Tanto las muestras clínicas obtenidas de sitios habitualmente estériles, como aquellas tomadas de sitio con microbiota comensal deberían ser inoculadas en un medio enriquecido como agar chocolate y en medio selectivo ${ }^{46,49,52}$.

En nuestro país, el medio selectivo de uso general es el agar Thayer-Martin, el cual contiene una base de agar nutritivo GC, adicionado de hemoglobina, un suplemento nutritivo que contiene numerosos factores de crecimiento y un suplemento antimicrobiano. No obstante, cepas de Neisseria lactamica y Kingella denitrificans pueden crecer con cierta frecuencia en agar Thayer Martin, mientras que otras especies comensales de Neisseria como N. subflava y $N$. cinerea crecen en forma ocasional ${ }^{52}$. Algunas cepas auxotróficas de $N$. gonorrhoeae, especialmente las cepas deficientes en arginina, hipoxantina y uracilo (auxotipo AHU) son susceptibles a las concentraciones habituales de vancomicina presentes en los medios selectivos ${ }^{52}$. Este hecho hace recomendable el efectuar control periódico del crecimiento de las cepas uretrales y endo-cervicales de una comunidad en medio selectivo y no selectivo ${ }^{52}$.

Identificación. El método tradicional de identificación bioquímica se basa en demostrar su patrón de utilización de azúcares. Neisseria gonorrhoeae oxida la glucosa, pero 
ningún otro azúcar de un panel que incluye lactosa, maltosa, sacarosa y fructosa. La demostración de utilización de azúcares en agar CTA no es recomendada ya que requiere un inóculo denso, larga incubación y puede dar resultados negativos por falta de crecimiento en el medio o por producción insuficiente de ácido de los carbohidratos ${ }^{52}$. Una modificación mejorada del procedimiento de utilización de azúcares en agar CTA consiste en el empleo de placas de agar $\mathrm{CTA}^{55}$, pero el método de elección es la prueba de utilización de azúcares rápida que no requiere crecimiento de la bacteria y que se basa en sus enzimas preformadas ${ }^{56}$. La detección de actividad prolil iminopeptidasa (PIP) mediante una prueba cromogénica, es un marcador fenotípico ampliamente utilizado en forma casera como incluido en pruebas comerciales. Sin embargo, la aparición de cepas PIP negativo ha invalidado la prueba. Alexander e Ison compararon siete métodos comerciales de identificación: cuatro bioquímicos, tres inmunológicos, y la prueba de utilización de azúcares en agar CTA en 100 cepas de $N$. gonorrhoeae. Los cuatro métodos bioquímicos evaluados fueron RapID NH® (Remel), API NH® (bioMérieux), Gonochek II ${ }^{\circledR}$ (E-Y Laboratorios) y Neisseria PET® (Key Scientific), los cuales identificaron solamente 64 a $66 \%$ de las cepas. Los ensayos utilizan la actividad PIP como marcador, detectándose $34 \%$ de cepas PIP negativo en el estudio ${ }^{57}$. Actualmente, con el mejoramiento de los métodos de identificación comercial para sistemas automatizados, la identificación de $N$. gonorrhoeae es superior, pero persisten problemas para la identificación de especies de Neisseria comensales ${ }^{58}$. La prueba de azúcares requirió repetir el $17 \%$ de las reacciones hasta tener resultados positivos ${ }^{57}$.

Existen varios métodos inmunológicos para la identificación de $N$. gonorrhoeae. Los más conocidos son MicroTrak $N$. gonorrhoeae culture confirmation test ${ }^{\circledR}$ (Trinity Biotech), que se basa en inmunofluorecencia; Phadebact monoclonal GC $®$ (Boule) que es una técnica de


se basa en inmunocromatografía. Los tres inmunoensayos utilizan AcMo que reconocen epitopos de ambos isotipos; PIA y PIB de la proteína PI de la membrana externa. Los tres procedimientos tienen 99 a $100 \%$ de sensibilidad y especificidad $^{46,57}$.

Técnicas de amplificación de los ácidos nucleicos. Las TAAN presentan, aún en la actualidad, limitaciones para el diagnóstico de $N$. gonorrhoeae, por lo que sus resultados son considerados presuntivos de infección ${ }^{26}$. Su principal debilidad es su falta de especificidad, derivada del empleo de blancos de amplificación no específicos, construidos sin el conocimiento de las secuencias genómicas de las neiserias comensales y también por el intercambio genético horizontal en el género Neisseria ${ }^{59-60}$. Entre los genes utilizados para diagnóstico de $N$. gonorrhoeae y en los que se ha observado zonas homólogas se cuentan 16S rARN y $c p p B^{60-61}$.

La existencia de falsos positivos es especialmente importante en poblaciones con baja prevalencia de infección, ya que disminuye considerablemente el valor predictor positivo de los ensayos ${ }^{62}$. Por este motivo, el CDC recomienda la confirmación de resultados positivos en poblaciones con baja prevalencia y en casos de abuso sexual $^{26}$.

Como ya se señalara, el CDC recomienda solamente el empleo de TAAN en muestras endo-cervicales, endouretrales y orina de hombres y esta recomendación se debe a que con el empleo de otras muestras clínicas aumenta la posibilidad de amplificar el ADN de especies comensales como $N$. lactamica y $N$. polysaccharea y de $N$. meningitidis debido la mayor abundancia de estas especies en estas muestras ${ }^{26,60}$.

Existen varias técnicas comerciales de amplificación, incluyendo Cobas Amplicor ${ }^{\circledR}$ (Roche), Gen-Probe APTIMA Combo 2® (Gen-Probe), Becton Dickinson Probe Tec assay ${ }^{\circledR}$ (Becton Dickinson) y Abbott Ligase Chain Reaction (Lcx)® (Abbott), además de numerosos protocolos caseros de RPC, que han sido revisados por Whiley y cols ${ }^{59}$. Uno de los sistemas comerciales más utilizados es Cobas Amplicor CT/NG® y en E.U.A., el fabricante recomienda repetir los valores de absorbancia $\left(\mathrm{A}_{450}\right)$ entre 0,2 y 3,5 por caer en la categoría de resultados equívocos hasta que, al menos 2 de 3 resultados, tenga una $\mathrm{OD} \geq 2,0$. Peter-Getzlaff y cols observaron que 258 de 398 muestras con OD > 0,2 (64,8\%) fueron negativas en tres ensayos de confirmación, mientras que las 140 muestras restantes $(35,2 \%)$ fueron confirmadas como positivas en al menos dos ensayos de confirmación ${ }^{61}$. La repetición de los resultados con OD en zona crítica aumenta la especificidad de las técnicas de amplificación y es especialmente importante en poblaciones con baja prevalencia de infección ${ }^{26,62}$. Sin embargo, la necesidad de repetir innumerables exámenes aumenta también considerablemente el costo y el riesgo de contaminación cruzada.

Entre los problemas derivados de la sensibilidad de esta técnica destaca la aparición y expansión geográfica temporal de cepas de $N$. gonorrhoeae que perdieron parte del blanco de amplificación, particularmente el gen сррB. Este gen se ubica en el plásmido críptico, 4,2 kb, de $N$. gonorrhoeae y puede experimentar recombinación interna generando cepas con una deleción parcial, lo que ha sido observado en varias regiones del mundo, pudiendo afectar hasta $10 \%$ de las cepas ${ }^{63}$. Recientemente se vivió en Suecia el mismo problema con la aparición de una variante genética de $C$. trachomatis que sufrió una deleción de 377 pb de su plásmido críptico, 7,2 kb y que incluyó precisamente el área blanco de amplificación de los TAAN comerciales COBAS Amplicor®, Taqman CT/ NG® y Abbott m2000rt CT/NG® ${ }^{8}$. Por este motivo, los 
centros de referencia debieran vigilar permanentemente en sus poblaciones la sensibilidad de las técnicas de amplificación en comparación con las técnicas de aislamiento de los microorganismos. Recientemente un estudio evaluó la utilidad de un ensayo de RPC en tiempo real con un doble blanco de amplificación, demostrando muy buena sensibilidad y especificidad incluyendo la detección de $N$. gonorrhoeae en muestras faríngeas, lo que sugiere que las técnicas de RPC múltiple podrían resolver los problemas actuales de las técnicas de amplificación para este microorganismo ${ }^{64}$.

\section{Trichomonas vaginalis}

\section{Muestras clínicas}

La muestra de elección para el diagnóstico de $T$. vaginalis por cultivo o TAAN en la mujer es la secreción vaginal, mientras que el rendimiento de la orina de primer chorro es $40 \%$ menos sensible ${ }^{65}$. Para el diagnóstico de infección masculina son apropiadas las muestras de orina, secreción uretral o semen, pero el mejor rendimiento se obtiene con la toma de más de un tipo de muestra lo que dificulta el diagnóstico ${ }^{24}$.

\section{Técnicas de diagnóstico}

Examen microscópico directo. El diagnóstico de $T$. vaginalis se efectúa, generalmente, por examen microscópico al fresco de secreción vaginal, uretral en hombres o de sedimento de orina de primer chorro. Este procedimiento tiene entre 36 y $70 \%$ de sensibilidad ya que depende de la capacidad de visualización de tricomonas móviles, lo que se relaciona con el tiempo de transporte de las muestras al laboratorio ${ }^{26,66}$. Las muestras deben ser observadas al microscopio en las primeras dos horas de obtenidas, ya que el microorganismo va perdiendo paulatinamente la movilidad. Por otra parte, el examen al fresco es un examen rápido, de bajo costo y buena especificidad y existe desinterés en implementar en los laboratorios clínicos en el mundo técnicas de mayor sensibilidad. Esto se explica en gran medida, porque existe en general desconocimiento de las implicancias que tiene la infección y ésta se detecta con baja frecuencia en los laboratorios clínicos. Al menos en Chile, la mayoría de los casos de tricomoniasis en la población femenina son diagnosticados clínicamente y tratados a nivel de los consultorios de atención primaria ${ }^{67}$.

Cultivo. Es la técnica de referencia para el diagnóstico de $T$. vaginalis, dadas su buena sensibilidad y excelente especificidad ${ }^{20,65,68}$. Existen varios medios de cultivo, pero el caldo Diamond modificado ha demostrado ser uno de los más sensibles y además existe en forma comercial (Remel, E.U.A.). El mayor rendimiento del cultivo se obtiene cuando las tórulas son depositadas de inmediato en el medio que ha alcanzado la temperatura ambiente, para luego ser enviados al laboratorio, también a temperatura ambiente ${ }^{68-69}$. No obstante, como se mencionara en neiserias, este procedimiento no resulta práctico. Beverly y cols, compararon la eficiencia del medio de transporte Amies conservado a temperatura ambiente por $24 \mathrm{~h}$ (Copan Diagnostic, Ca, E.U.A.) con la siembra directa de muestras clínicas de 260 pacientes $^{70}$. En 68 (26\%) muestras positivas, la sensibilidad de la inoculación directa fue $94,1 \%$ y la del uso de medio de transporte, $91,2 \%$, diferencias que no fueron significativas ${ }^{70}$. Desafortunadamente, no se han efectuado otros estudios con otros medios de transporte habituales para confirmar los resultados anteriores.

Los medios de cultivo para tricomonas contienen digeridos enzimáticos, habitualmente de caseína e hígado, extracto de levadura, dextrosa o maltosa y 5 a $10 \%$ de suero de caballo o bovino. Contienen también una pequeña cantidad de agar, el que limita la difusión del $\mathrm{O}_{2}$ al interior y cisteína- $\mathrm{HCl}$ como agente reductor, ya que $T$. vaginalis es un organismo anaerobio. Para inhibir la microbiota acompañante, se emplean antimicrobianos, elegidos entre penicilina, gentamicina o vancomicina y como fungicida, anfotericina B. Trichomonas. vaginalis requiere un $\mathrm{pH}$ óptimo de 6,0 a 6,3 , mientras que otras especies crecen mejor a $\mathrm{pH}$ neutro ${ }^{71}$.

Los cultivos son examinados a las 24, 48 y 96 hs de incubación en aerobiosis a $36^{\circ} \mathrm{C}$ y, si están negativos, se hace una última lectura a los 7 días. Sin embargo, la mayoría de los cultivos están positivos entre las 24 y 48 hs de incubación ${ }^{68}$. La lectura de los medios se efectúa mediante examen al fresco de una gota obtenida desde el fondo del caldo, con objetivo de 10X. Esto permite enfocar un campo visual más amplio y detectar rápidamente la movilidad de los microorganismos. Los cultivos positivos frescos muestran una gran cantidad de células de vigorosa movilidad y morfología característica. Con el paso de los días, las células se redondean, pierden la capacidad de desplazamiento y sólo conservan la movilidad de los flagelos anteriores, hasta que finalmente se lisan.

Las dificultades del cultivo son la necesidad de refrigerar los medios a $4{ }^{\circ} \mathrm{C}$ y su menor tiempo de expiración, dados por la inactivación de los antimicrobianos y de la difusión paulatina del oxígeno. No es fácil conservar cepas de referencia o cepas clínicas para control. Las cepas pueden ser conservadas en continuo sub-cultivo en medio fresco. Los microorganismos se mueren a $20{ }^{\circ} \mathrm{C} \mathrm{y}$ su conservación a $-70{ }^{\circ} \mathrm{C}$ no es tampoco segura.

Técnicas moleculares. La FDA no ha aprobado aún procedimiento comercial alguno de amplificación de los ácidos nucleicos, pero existen numerosas RPC caseras $^{26}$. Los partidores empleados para diagnóstico de T. vaginalis han sido bien analizados y no se han detectado falsos positivos frente a otras especies de Trichomonas u otros 
protozoos. Estudios en los que se ha utilizado como técnica de referencia el cultivo o la confirmación de RPC por una segunda RPC y en los que se ha incluido un número significativo de pacientes, han determinado que la sensibilidad de la RPC para T. vaginalis varía entre 84 y 97\%, con valores de especificidad de 94 a $98 \%$ en muestras de secreción vaginal ${ }^{65-66,72}$. La sensibilidad y especificidad en orina son 64,2 y $100 \%$ respectivamente ${ }^{65}$.

\section{Otras técnicas de diagnóstico}

Las técnicas inmunológicas clásicas de EIA e IFD tienen menor sensibilidad que el cultivo o técnicas moleculares, por lo que su implementación no tiene justificación ${ }^{68}$. Se dispone también de algunas técnicas inmunocromatográficas rápidas como OSOM Trichomonas rapid test ${ }^{\circledR}$ y Xenostrip-Tv $\AA$, pero su sensibilidad, aunque superior a la del examen al fresco, es inferior a la sensibilidad del cultivo y o RPC y tienen resultados falsamente positivos en poblaciones con baja prevalencia de infección, por lo que no se recomienda su uso en los laboratorios ${ }^{26,73}$.

El diagnóstico de tricomonas mediante la tinción de Papanicolaou tiene la ventaja de su amplia disponibilidad y su buena especificidad, $95 \%{ }^{74}$. Sin embargo, la tinción tiene alrededor de 40 a $60 \%$ de falsos negativos ${ }^{74,75}$. Las tricomonas suelen redondearse y perder su morfología piriforme en muestras con alto contenido en leucocitos, lo que dificulta distinguirlas de otros elementos celulares.

\section{Conclusiones}

Las evidencias actualmente disponibles indican que, en virtud de su buena sensibilidad y especificidad, el cultivo debería continuar siendo la técnica de referencia para el diagnóstico de $N$. gonorrhoeae. El cultivo tiene además la ventaja de ser apropiado para cualquier tipo de muestras y permite recolectar cepas para la vigilancia de su susceptibilidad antimicrobiana. Para el diagnóstico de C. trachomatis, las TAAN tienen excelente sensibilidad y especificidad, permitiendo detectar hasta $30 \%$ más de muestras positivas que otros procedimientos y no requieren de una toma de muestra invasora. Esto los convierte en las técnicas de referencia para diagnóstico, especialmente en mujeres, que suelen ser asintomáticas. Es necesario implementar en Chile las normas de diagnóstico en todas sus etapas y un sistema de control de los procedimientos de laboratorio de C. trachomatis. Finalmente, el cultivo es la técnica de referencia para T. vaginalis, no justificándose la implementación de ensayos de TAAN, que son más costosos y complejos.

\section{Resumen}

Las infecciones de transmisión sexual (ITS) no virales son causa de sufrimiento físico, psicológico y social, tienen consecuencias graves para la salud reproductiva de la mujer y pueden ser transmitidas al recién nacido. Estas infecciones son también un factor de riesgo de adquirir y transmitir otras ITS y en la mujer embarazada están asociadas con riesgo de parto prematuro. En los últimos años se ha producido una declinación en las tasas nacionales de gonorrea, pero es necesario implementar el tamizaje de Chlamydia trachomatis, dado que hasta el $80 \%$ de las infecciones por esta bacteria son asintomáticas. Por razones médicas, psicosociales y legales, el diagnóstico de laboratorio de las ITS debe ser de certeza, lo que plantea un desafío al laboratorio, ya que la labilidad de los agentes, su gran adaptación al hospedero humano y las particularidades de su fisiología hacen que su diagnóstico presente mayor dificultad que el de otras bacterias. Por otra parte, las técnicas de laboratorio disponibles actualmente para el diagnóstico de las ITS no virales se caracterizan por su excelente sensibilidad y especificidad, lo que resulta muy valioso dada la naturaleza curable de estas infecciones. El diagnóstico de ITS y de infecciones genitales asociadas como candidiasis vulvovaginal y vaginosis bacteriana representa una importante fracción de la demanda de diagnóstico en los laboratorios clínicos. Por este motivo, el establecimiento de normas y controles de calidad de los laboratorios que efectúan diagnóstico de estas infecciones, como asimismo la vigilancia epidemiológica y genética de agentes de transmisión sexual debería ser considerado una prioridad en nuestro país. Esta revisión se centra en tres microorganismos de transmisión sexual no virales; C. trachomatis, Neisseria gonorrhoeae y Trichomonas vaginalis y tiene como objetivos actualizar las evidencias que justifican la implementación de técnicas de diagnóstico eficientes para estos agentes y mostrar los avances producidos para su diagnóstico microbiológico. 


\section{Referencias}

1.- Ovalle A, Martínez M A. Infecciones genitales. E. Guzmán (Editor). Selección de temas en Gineco-obstetricia. Tomo II. Publiimpacto 2008; p. 875-923.

2.- Comité Normas Infecciones de Transmisión Sexual. Ministerio de Salud de Chile. Normas de manejo y tratamiento de infecciones de transmisión sexual (ITS). Primera parte. Rev Chil Infectol 2009; 26: 174-90.

3.- World Health Organization. Global prevalence and incidence of selected curable sexually transmitted infections, overview and estimates. WHO/CDS/EDC/2001.10.Geneva, 2001.

4.- Romoren M, Velauthapillai M, Rahman M, Sundby J, Klouman E, Hjortdahl P. Trichomoniasis and bacterial vaginosis in pregnancy: inadequately managed with the syndromic approach. Bull World Health Org 2007; 85: 297-304.

5.- Peeling R W, Mabey D, Herring A, Hook $3^{\text {rd }}$ EW. Why do we need quality-assured diagnostic tests for sexually transmitted infections?. Nat Rev Microbiol 2006; 4: 909-20.

6.- Lum G, Freeman K, Limnios E A, Tabrizi S N, Carter I, Chambers I W, et al. A cluster of culture positive gonococcal infections but with false negative $c p p B$ gene based PCR. Sex Transm Infect 2005; 81: 400-2.

7.- Alexander S, Martin I M, Fenton K, Ison C A. The prevalence of praline iminopeptidase negative Neisseria gonorrhoeae throughout England and Wales. Sex Transm Inf 2006; 82: 280-2.

8.- $\quad$ Ripa T, Nilsson P A. A Chlamydia trachomatis strain with a 377-bp deletion in the cryptic plasmid causing false negative nucleic acid amplification tests. Sex Transm Dis 2007; 34: 255-6.

9.- $\quad$ Morré S A, Van den Brule A J, Rozendaal L, Boeke A J, Voorhorst F J, De Blok S, et al. The natural course of asymptomatic Chlamydia trachomatis infections: $45 \%$ clearance and no development of clinical PID after one-year follow-up. Int J STD\& AIDS 2002; 13 (Suppl 2): $12-8$.

10.- Hillis S D, Wasserheit I. Screening for chlamydia -a key to the prevention of pelvic inflammatory disease. N Engl J Med 1996; 334 : 1399-401.

11.- Ovalle A, Martínez M A, Casals A, Yuhaniak R, Giglio M S. Estudio clínico y microbiológico de la enfermedad inflamatoria pélvica aguda. Rev Chil Obstet Ginecol 1993; 58: 103-12.

12.- Dereli D, Coker M, Ertem E, Serter D, Tanac R, Tez E. Chlamydial infections in infants. J Trop Pediatr 1996; 42: 233-6.

13.- Chawla R, Bhalla P, Sachdev H P. A pilot study of Chlamydia trachomatis pneumonia in infants. Indian J Med Microbiol 2004; 3: 185-7.

14.- Rosenman M B, Mahon B E, Downs S M, Kleiman M B. Oral erythromycin prophylaxis $v s$ watchful waiting in caring for newborns exposed to Chlamydia trachomatis. Arch Pediatr Adolesc Med 2003; 157: 565-71.

15.- Valencia C, Prado V, Ríos M, Cruz M A, Pilorget J J. Prevalencia de Chlamydia trachomatis en conjuntivitis neonatal determinada mediante las técnicas de inmunofluorescencia y amplificación génica. Rev Méd Chile 2000; 128: 758-65.

16.- Martínez M A, Millán F, González F. Chlamydia trachomatis genotypes associated with pneumonia in Chilean infants. Scand J Infect Dis 2009; 41: 313-6.

17.- Stern A, Brown M, Nickel P, Meyer T F. Opacity genes in Neisseria gonorrhoeae: control of phase and antigenic variation. Cell 1986; 47 : 61-71.

18.- Edwards J L, Apicella M A. The molecular mechanisms used by Neisseria gonorrhoeae to initiate infection differ between men and women. Clin Microbiol Rev 2004; 17: 965-81.

19.- Tauber P F, Wettich W, Nohlen M, Zaneveld L J. Diffusible proteins of the mucosa of the human cervix, uterus, and fallopian tubes: distribution and variations during the menstrual cycle. Am J Obstet Gynecol 1985; 151: 1115-25.

20.- Petrin D, Delgaty K, Bhatt R, Garber G. Clinical and microbiological aspects of Trichomonas vaginalis. Clin Microbiol Rev 1998; 11: 300-17.

21.- Minkoff H, Grunebaum A N, Schwarz R H, Feldman J, Cummings M C, Clark W L, et al. Risk factors for prematurity and premature rupture of membranes: a prospective study of the vaginal flora in pregnancy. Am J Obstet Gynecol 1984; 150: 965-72.

22.- Cotch M F, Pastorek J G, Nugent R P, Hillier S L, Gibbs R S, Martin D H, et al. Trichomonas vaginalis associated with low birth weight and preterm delivery. Sex Transm Dis 1997; 24: 361-2.

23.- Wendel K A, Erbelding E J, Gaydos C A, Rompalo A M. Use of urine polymerase chain reaction to define the prevalence and clinical presentation of Trichomonas vaginalis in men attending an STD clinic. Sex Transm Infect 2003; 79: 151-3.

24.- Hobbs M M, Lapple D M, Lawing LF, Schwebke J R, Cohen M S, Swygard H, et al. Methods for detection of Trichomonas vaginalis in the male partners of infected women: implications for control of trichomoniasis. J Clin Microbiol 2006; 44: 3994-9.

25.- Laga M A, Manoka A, Kivuvu M, Malele B, Tuliza M, Nzila N, et al. Non-ulcerative sexually transmitted diseases as risk factors for HIV-1 transmission in women: results from a cohort study. AIDS 1999; 7: 95-102.

26.- Centers for Disease Control and Prevention. Screening tests to detect Chlamydia trachomatis and Neisseria gonorrhoeae infections- 2002. MMWR Morb Mortal Wkly Rep 2002/51 (RR15): 1-27.
27.- Watson E J, Templeton A, Russell I, Paavonen J, Mardh P A, Stary A, et al. The accuracy and efficiency of screening tests for Chlamydia trachomatis: a systematic review. J Med Microbiol 2002; 51: 1021-31.

28.- Shafer M A, Moncada J, Boyer C B, Betsinger K, Flinn S D, Schachter J. Comparing first-void urine specimens, self-collected vaginal swabs, and endocervical specimens to detect Chlamydia trachomatis and Neisseria gonorrhoeae by a nucleic acid amplification test. J Clin Microbiol 2003; 41: 4395-9.

29.- Schachter J, Chernesky M A, Willis DE, Fine P M, Martin D H, Fuller D, et al. Vaginal swabs are the specimens of choice when screening for Chlamydia trachomatis and Neisseria gonorrhoeae: results from a multicenter evaluation of the APTIMA assays for both infections. Sex Transm Dis 2005; 32: 725-8.

30.- Black C M. Current methods of laboratory diagnosis of Chlamydia trachomatis infections. Clin Microbiol Rev 1997; 10: 160-84.

31.- Battle T J, Golden M R, Suchland K L, Counts J M, Hughes J P, Stamm W E, et al. Evaluation of laboratory testing methods for Chlamydia trachomatis infection in the era of nucleic acid amplification. J Clin Microbiol 2001; 39 : 2924-7.

32.- Phillips R S, Hanff P A, Kaufman R S, Aronson M D. Use of a direct fluorescent antibody test for detecting Chlamydia trachomatis in women seeking routine gynecologic care. J Infect Dis 1987; 156: 578-81.

33.- Lefebvre J, Laperriere H, Rousseau H, Masse R. Comparison of three techniques for detection of Chlamydia trachomatis in endocervical specimens from asymptomatic women. J Clin Microbiol 1988; 26: 726-31.

34.- Schachter J, Jones R B, Butler R C, Rice B, Brooks D, Van der Pol E. Evaluation of the Vidas Chlamydia test to detect and verify Chlamydia trachomatis in urogenital specimens. J Clin Microbiol 1997; 35: 2102-6.

35.- Waites K B, Smith K R, Crum M A, Hockett R D, Wells A H, Hook III E W. Detection of Chlamydia trachomatis endocervical infections by ligase chain reaction versus ACCESS Chlamydia antigen assay. J Clin Microbiol 1999; 37: 3072-3.

36.- Newhall W J, Johnson R E, DeLisle S, Fine D, Hadgu A, Matsuda B, et al. Head-to-head evaluation of five chlamydia tests relative to a quality-assured culture standard. J Clin Microbiol 1999; 37: 681-5.

37.- Lauderdale T-L, Landers L, Thorneycroft I, Chapin K. Comparison of PACE2 assay, two amplification assays, and Clearview EIA for detection of Chlamydia trachomatis in female endocervical and urine specimens. J Clin Microbiol 1999; 37: 2223-9.

38.- Shafer M A, Schachter J, Moncada J, Keogh J, Pantell R, Gourlay L. Evaluation of urine-based 
screening strategies to detect Chlamydia trachomatis among sexually active asymptomatic young males. JAMA 1993; 270: 2065-71.

39.- Gift T L, Pate M S, Hook $3^{\text {rd }}$ E W, Kassler W $\mathrm{J}$. The rapid test paradox: when fewer cases detected lead to more cases treated: a decision analysis of tests for Chlamydia trachomatis. Sex Transm Dis 1999; 26: 241-2.

40.- Swain G R, McDonals R A, Pfister J R, Gradus S, Sedmak G V, Singh A. Decision analysis: point-of-care Chlamydia testing vs laboratory methods. Clin Med Res 2004; 2 : 29-35.

41.- Bébéar C, De Barbeyrac B. Genital Chlamydia trachomatis infections. Clin Microbiol Infect 2009; 15: 4-10.

42.- Gaydos C A, Ferrero D V, Papp J. Laboratory aspects of screening men for Chlamydia trachomatis in the new millennium. Sex Transm Dis 2008; 35: S45-50.

43.- Black C M, Marrazzo J, Johnson R E, Hook E W $3^{\text {rd }}$, Jones R B, Green T A, et al. Head-tohead multicenter comparison of DNA probe and nucleic acid amplification tests for Chlamydia trachomatis infection in women performed with an improved reference standard. J Clin Microbiol 2002; 40: 3757-63.

44.- Schachter J, Chow J M, Howard H, Bolan G, Moncada J. Detection of Chlamydia trachomatis by nucleic acid amplification testing: our evaluation suggests that CDC-recommended approaches for confirmatory testing are illadvised. J Clin Microbiol 2006; 44: 2512-7.

45.- Schachter J, Hook E W, Martin D H, Willis D, Fine P, Fuller D, et al. Confirming positive results of nucleic acid amplification tests (NAATs) for Chlamydia trachomatis: all NAATs are not created equal. J Clin Microbiol 2005; 43: 1372-3.

46.- Lai-King N G, Martin I E. The laboratory diagnosis of Neisseria gonorrhoeae. Can J Infect Dis Med Microbiol 2005; 16: 15-25.

47.- Van Horn K G, Audette C D, Sebeck D, Tucker K A. Comparison of the Copan ESwab system with two Amies agar swab transport systems for maintenance of microorganism viability. J Clin Microbiol 2008; 46: 1655-8.

48.- Olsen C C, Schwebke J R, Benjamin W H, Beverly A, Waites K B. Comparison of direct inoculation and Copan transport systems for isolation of Neisseria gonorrhoeae from endocervical specimens. J Clin Microbiol 1999; 37: 3583-5.

49.- Janda W M, Knapp J S. Neisseria and Moraxella catarrhalis. Murray P R, Baron E J, Jorgensen J H, Pfaller M A, Yolken R H, editors. Manual of Clinical Microbiology, 8th ed. Washington, DC: American Society for Microbiology 2000; p. 585-608.
50.- Clinical and Laboratory Standard Institute. Quality control of microbiological transport systems. Approved standard 2003. M40-A. CLSI, Wayne, PA.

51.- Graver M A, Wade J J. Survival of Neisseria gonorrhoeae isolates of different auxotypes in six commercial transport systems. J Clin Microbiol 2004; 42: 4803-4.

52.- Knapp J S, Rice R J. Neisseria and Branhamella. Murray P R, Baron E J, Pfaller M A, Tenover F C, Yolken R H, editors. Manual of Clinical Microbiology, $6^{\text {th }}$ ed. Washington DC: American Society for Microbiology 1995; p. 324-40.

53.- Vern-Juchau S, Nackman R, Ruppart D. Comparison of Gram stain with DNA probe for detection of Neisseria gonorrhoeae in urethras of symptomatic males. J Clin Microbiol 1995; 33: 3068-9.

54.- Bignell C, Ison C A, Jungmann E. Gonorrhoea. Sex Transm Infect 2006; 82 (Suppl 4): iv6-9.

55.- Taylor G S, Keys R J. Simplified sugar fermentation plate technique for identification of Neisseria gonorrhoeae. Appl Microbiol 1974; 27: 416-7.

56.- Pollock H M. Evaluation of methods for the rapid identification of Neisseria gonorrhoeae in a routine clinical laboratory. J Clin Microbiol 1976; 4: 19-21.

57.- Alexander S, Ison C. Evaluation of commercial kits for the identification of Neisseria gonorrhoeae. J Med Microbiol 2005; 54 : 827-31.

58.- Rennie R P, Brosnikoff C, Shokoples S, Reller L B, Mirrett S, Janda W, et al. Multicenter evaluation of the new Vitek 2 NeisseriaHaemophilus identification card. J Clin Microbiol 2008; 46: 2681-5.

59.- Whiley D M, Tapsall J W, Sloots T P. Nucleic acid amplification testing for Neisseria gonorrhoeae. An ongoing challenge. J Mol Diagn 2006; 8: 3-15.

60.- Mangold K A, Regner M, Tajuddin M, Tajuddin A M, Jennings I, Du H, et al. Neisseria species identification assay for the confirmation of Neisseria gonorrhoeae-positive results of the COBAS Amplicor PCR. J Clin Microbiol 2007; 45: 1403-9.

61.- Peter-Getzlaff S, Luethy J, Springer B. Diagnostic value of molecular confirmation assays for Neisseria gonorrhoeae. J Clin Microbiol 2007; 45: 3856-8.

62.- Katz A R, Effler P V, Ohye R G, Brouillet B, Veneranda M, Lee C, et al. False-positive gonorrhoea test results with a nucleic acid amplification test: the impact of low prevalence on positive predictive value. Clin Infect Dis 2004; 38: 814-9.

63.- Lum G, Freeman K, Limnios E A, Tabrizi S N,
Carter I, Chambers I W, et al. A cluster of culture positive gonococcal infections but with false negative $c p p B$ gene based PCR. Sex Transm Infect 2005; 81: 400-2.

64.- Goire N, Nissen M D, LeCornec G M, Sloots T P, Whiley D M. A duplex Neisseria gonorrhoeae real-time polymerase chain reaction assay targeting the gonococcal porA pseudogene and multicopy opa genes. Diagn Microbiol Infect Dis 2008; 61: 6-12.

65.- Lawing L F, Hedges S R, Schwebke J R. Detection of trichomonosis in vaginal and urine specimens from women by culture and PCR. J Clin Microbiol 2000; 38: 3585-8.

66.- Madico G, Quinn T C, Rompalo A, Mc Kee K T, Gaydos C A. Diagnosis of Trichomonas vaginalis infection by PCR using vaginal swab samples. J Clin Microbiol 1998; 36: 3205-10

67.- Neira P, Correa A, Muñoz N, Tardio M T, Carabelli M. Frecuencia de infección por Trichomonas vaginalis en atención primaria de salud. Rev Chil Obstet Ginecol 2005; 70: 147-51.

68.- Patel R S, Wiese W, Patel S C, Ohl C, Byrd J C, Estrada C A. Systematic review of diagnostic tests for vaginal trichomoniasis. Infect Dis Obstet Gynecol 2000; 8: 248-57.

69.- Gelbart S M, Thomason J L, Osypowski P J, James J A, Hamilton P R. Comparison of Diamond's medium modified and Kupferberg medium for detection of Trichomonas vaginalis. J Clin Microbiol 1989; 27: 1095-6.

70.- Beverly A L, Venglarik M, Cotton B, Schwebke J R. Viability of Trichomonas vaginalis in transport medium. J Clin Microbiol 1999; 37: 3749-50.

71.- Diamond L S. In vitro cultivation of the Trichomonadidae: a state of the art review. Acta Universitates Carolinae-Biologica 1986; 30: 221-8.

72.- Wendel K A, Erbelding E J, Gaydos C A, Rompalo A M. Trichomonas vaginalis polymerase chain reaction compared with standard diagnostic and therapeutic protocols for detection and treatment of vaginal trichomoniasis. Clin Infect Dis 2002; 35: 576-80.

73.- Pillay A, Lewis J, Ballard R C. Evaluation of Xenostrip-Tv, a rapid diagnostic test for Trichomonas vaginalis infection. J Clin Microbiol 2004; 42: 3853-6.

74.- Wiese W, Patel S R, Patel S C, Ohl C A, Estrada C A. A meta-analysis of the Papanicolaou smear and wet mount for the diagnosis of vaginal trichomoniasis. Am J Med 2000; 108: 301-8.

75.- Thomason J L, Gelbart S M, Sobun J F, Schulien M B, Hamilton P R. Comparison of four methods to detect Trichomonas vaginalis. J Clin Microbiol 1988; 26: 1869-70. 